\title{
Healthcare Professionals and Students' Awareness of Chagas Disease: Design and Validation of Chagas Level of Knowledge Scale (ChaLKS)
}

\author{
José M. Ramos-Rincón,, ${ }^{1,2}$ José J. Mira-Solves, ${ }^{3,4}$ Violeta Ramos-Sesma, ${ }^{5}$ Diego Torrús-Tendero, ${ }^{2,6}$ Jara Llenas-García, ${ }^{7}$ \\ and Miriam Navarro ${ }^{8 \star}$
}

${ }^{1}$ Department of Clinical Medicine, Universidad Miguel Hernández de Elche, Alicante, Spain; ${ }^{2}$ Department of Internal Medicine, Hospital General Universitario de Alicante, Instituto de Investigación Sanitaria y Biomédica de Alicante (ISABIAL-Fundación FISABIO), Alicante, Spain; ${ }^{3}$ Department of Health Psychology, Universidad Miguel Hernández de Elche, Alicante, Spain; ${ }^{4}$ Alicante-Sant Joan Health District, Alicante, Spain; ${ }^{5}$ Department of Internal Medicine, Hospital Universitario de Torrevieja, Alicante, Spain; ${ }^{6}$ Parasitology Area, Universidad Miguel Hernández de Elche, Alicante, Spain; ${ }^{7}$ Department of Internal Medicine, Hospital Vega Baja, Fundación para el Fomento de la Investigación Sanitaria y Biomédica de la Comunitat Valenciana (FISABIO), Orihuela, Spain; ${ }^{8}$ Department of Public Health, Science History and Gynecology, Universidad Miguel Hernández de Elche, Alicante, Spain

\begin{abstract}
There are few studies evaluating awareness of Chagas disease among healthcare professionals attending migrants from Latin America or working in Chagas-endemic areas. The objective of this study was to design and validate instruments for assessing knowledge about Chagas disease among healthcare students and residents as well as students and professionals of social and other health science disciplines. Two validated scales have been developed: the 10-item Chagas Level of Knowledge Scale for healthcare professionals (ChaLKS-Medical) and the 8-item ChaLKSSocial\&Health for potential aid workers from those fields. Both scales were considered adequate in terms of readability, internal consistency, construct validity, and discriminant validity. The mean number of correct answers on the ChaLKSMedical among respondents from non-healthcare versus healthcare sectors was 1.80 versus $7.00(P<0.001)$. The scores on the ChaLKS-Social\&Health also discriminated between the knowledge levels in these two groups (1.76 versus 6.78 , $P<0.001)$. Knowledge among medical/pharmacy students and residents on the ChaLKS-Medical was acceptable and different (mean: 5.8 and 7.4, respectively; $P<0.001$ ). Respondents' knowledge of Chagas disease was greater in those who had previously received information on the disease; this was true in both respondents from the healthcare sector (mean correct answers, ChaLKS-Medical: 7.2 versus $4.3, P<0.001$ ) and in potential aid workers (mean correct answers, ChaLKS-Social\&Health: 5.1 versus $1.1, P=0.001$ ). The metric properties of both scales are adequate for their use in supporting aid operations in Chagas-endemic countries or in providing health and social care to migrant populations in non-endemic countries.
\end{abstract}

\section{INTRODUCTION}

Validation scales play an important role in developing accurate tools for measuring different phenomena. Established methods can examine the effect of certain factors through distinct validation steps, including content validation and checks for internal consistency, reproducibility, and criterion validity. The validated result guarantees the instrument's reliability, enabling its use in later studies. Several articles describe the development and validation of instruments to assess providers' knowledge of diseases, such as sickle cell disease, and to assess the health professionals' attitudes toward people affected by diseases. ${ }^{1,2}$

Chagas disease (CD) is a parasitic (Trypanosoma cruzi), mainly vector-borne, disease with a high public health impact in Europe, where other transmission routes (blood transfusion, transplantation, and mother-to-child) may also occur and are not completely controlled. ${ }^{3}$ Rising immigration from Latin America to Europe and especially to Spain has led to an increase in cases, mainly in asymptomatic immigrants in the chronic phase of the disease. ${ }^{4}$ However, CD is still largely unrecognized in Europe, and fewer than 10\% of cases are diagnosed. ${ }^{5}$ This has led to active efforts to identify patients to bring them into the healthcare system. ${ }^{6}$

Underdiagnosis of CD in Europe and other non-endemic regions has three main component causes, related to the

\footnotetext{
*Address correspondence to Miriam Navarro, Department of Public Health, Science History and Gynecology, Universidad Miguel Hernández de Elche. Crta. Nacional, N-332, s/n, 03550 Sant Joan,
} Alicante, Spain. E-mail: miriamtropical@gmail.com population at risk (lack of knowledge and awareness about the disease, fear, stigma, and barriers to access the healthcare system); healthcare professionals (lack of training in tropical medicine, global health, and cultural diversity in the consultation); and public health measures (so far insufficient to address the challenge of detecting and controlling this emerging neglected tropical disease [NTD]). ${ }^{3,7}$

There are few studies evaluating the awareness of $C D$ among healthcare professionals in Europe, ${ }^{8,9}$ and little is known about medical students' knowledge of the issue. ${ }^{10,11}$ Overall, these studies show room for improvement with regard to health professionals' knowledge of CD. Surveys in the United States-the country with the highest number of migrants infected with $T$. cruzi-also show a general lack of awareness of CD among physicians across specialties. ${ }^{12-14}$ Studies have indicated that U.S. physicians may not consider CD when diagnosing immigrant patients from Chagasendemic areas. ${ }^{7}$ Similar results have been reported in patients and healthcare professionals in Spain and other European countries. ${ }^{5,15}$

At the same time, international aid work in Latin America has changed drastically in the past decade, also affecting social organizations. This change is also relevant from the Spanish perspective. ${ }^{16,17}$ However, similar to the health sector, there are scarce data about aid workers' and volunteers' knowledge of CD before working with populations in or from endemic regions.

Having a validated scale to assess the level of knowledge of CD among healthcare professionals and students would help us to improve and adapt the educational plans for future healthcare professionals in the context of a globalized and changing world. 
A valid scale would also enable evaluation of training outcomes. Moreover, non-governmental organizations (NGOs) could use such a tool to evaluate the knowledge of CD among their volunteers and help them to use integrated approaches while designing and implementing their programs and interventions.

The objective of this study was to design and validate instruments for assessing knowledge about CD among medical and pharmacy students and residents, and among students of other health science and social disciplines who are interested in international cooperation in Latin America or in attending to Latin American migrants. A secondary aim was to assess the influence of having previous information about CD on the responses shown in the scale.

\section{MATERIALS AND METHODS}

Study design. The cross-sectional study conducted in Alicante, Spain, between January 2016 and March 2018, focused on the validation of both scales.

Participants. We recruited a convenience sample of 349 participants (66 students in fields other than medicine or pharmacy who were interested in aid work, plus 283 medicine and pharmacy students, recent medical school graduates and residents, and recent pharmacy school graduates and residents). The sample size was determined to detect a difference of 0.39 points, with a confidence level of $95 \%$. The inclusion criteria were as follows: medicine and pharmacy students, students and professionals from other disciplines related to aid work who had obtained their bachelor's degree in Europe, and doctors, pharmacists, and other health professionals who had recently completed or were completing their specialty training in Spain. Participants were recruited through six events or channels (conferences or classes on aid work, etc. Supplemental Table 1) and replied using a self-administered questionnaire (pencil-paper and online approaches were used). All participants were informed about the objective of the study before completing the survey.

To perform the study, a reactive questionnaire (which includes the items that initially form part of the pool of questions that are going to be analyzed) was designed to assess the respondents' level of knowledge. The tool was analyzed for readability, reliability, and validity.

Scale design. Two authors developed an initial set of questions based on a prior questionnaire, widely used in Madrid (Spain), aimed at populations at risk of CD and described in a report published by the Spanish Ministry of Health. ${ }^{18}$ This questionnaire contained 26 reactive items, encompassing knowledge of Chagas epidemiology (five items), transmission routes (eight items), clinical characteristics (six items), diagnosis (four items), and treatment (three items) (see Supplemental Table 2). This proposal was assessed for relevance, clarity, and priority by three internists, three family and community medicine specialists (all with experience in CD), and three physicians from Latin America with knowledge of CD, to best discriminate between different levels of knowledge (face and content validity analyses). These experts ranked the importance of the items and recommended eliminating three. Following this analysis, our team agreed to design a test on CD directed toward medical and pharmacy students and another directed toward students in other health and social fields who were interested in international aid work in areas with population at risk of CD.
There are a significant number of volunteers and staff with no specific global health training who join international cooperation projects in Chagas-endemic regions or collaborate with local NGOs devoted to refugees and migrants from these countries. For this reason, we adapted the scale to this group, retaining items related to basic knowledge about CD transmission routes and excluding those related to diagnosis and treatment $(n=8)$. Based on our experience (both in endemic and non-endemic countries) and our literature review, we selected the items testing the knowledge needed for protecting oneself, avoiding the stigmatization of the affected population, and determining the need for additional training before joining a project/intervention. The items finally selected for the ChaLKS-Social\&Health questionnaire focus on CD epidemiology and transmission.

The version for healthcare students and residents included 23 items, whereas the one for potential aid workers had 15 items (Supplemental Table 3a in Spanish and Supplemental Table 3b in English).

All questions for both questionnaires included three possible responses: "yes," "no," and "I don't know." The scores in both questionnaires were the sum of the correct answers; incorrect answers were not penalized. One of the questions on transmission was initially formulated as an open question ("What insect spreads Chagas disease?"). The four most frequent answers were mosquito, sandfly, fly, and kissing bug (Supplemental Table 2). In the final version of the questionnaire, the item was reformulated as closed to maintain the same format as for the other items ("Do kissing bugs spread Chagas disease?") (Supplemental Table 5a and $b)$.

To assess comprehension and the potential for misunderstandings, we asked three third-year students in occupational therapy, podiatry, and physical therapy; three last-year medical students; and three last-year pharmaceutical students to read the respective versions of the questionnaire for aid workers and health professionals. The questionnaire was piloted among these 15 students.

The difficulty index applied in this study followed the standard formula: the difficulty index of each question was calculated by dividing the number of correct responses by the number of total respondents; higher scores corresponded to easier items. We deemed indexes of $0.50-0.60$ to be an average level of difficulty, indexes of $0.31-0.49$ to be a moderate level of difficulty, and we also aimed to include $5 \%$ of questions with a high level of difficulty (less than 0.30 ).

Reliability. Cronbach's alpha was applied as a measure of internal consistency. Reliability was calculated using the splithalf method, applying the Spearman-Brown coefficient. A minimum of 0.70 was considered acceptable for both statistics. The item-total correlation was also analyzed to characterize the metric properties of the elements, excluding those elements with low correlations. A minimum coefficient of 0.35 for Pearson's correlation was considered acceptable.

Construct validity. An exploratory factor analysis (EFA) was performed to determine the factorial structure of each instrument using the principal components technique, followed by the varimax rotation. A factor loading greater than 0.5 was considered an acceptable level of missing data. Before this analysis, the Kaiser-Meyer-Olkin measure of sampling adequacy was applied to identify the proportion of variance in the items that might be caused by underlying factors. Also, 
Bartlett's test of sphericity was applied to determine whether the EFA was suitable for structure detection.

Discriminant validity. The responses from the two subsamples were compared to check that healthcare students and resident physicians consistently obtained better scores than non-healthcare students (discriminant validity). Using the Student's $t$-test, we compared the number of correct answers obtained by respondents who reported having versus not having received prior training in CD with those who had received their bachelor's degree in Latin America versus Europe. We also compared the performance of different subgroups: medicine and pharmacy students along with medical and pharmacy residents. Finally, we compared the scores of potential aid workers and students, and medical and pharmacy professionals. Scales should demonstrate they are able to discriminate the level of knowledge between groups of participants. In this case, it was tested if two measures that should be different were actually different and that these differences were going in the expected direction.

Translation. A certified Spanish to English translator with expertise in scientific documents translated the scales from Spanish. A forward-backward translation was then performed by another independent translator to obtain a Spanish version of these instruments (from the English-language ones), to check their similarity.

Analysis statistics. Categorical data were expressed as absolute and relative frequencies. The items for both scales were compared by the type of respondent by means of chi-squared tests and Fisher's exact test (in the case of small numbers). Quantitative variables were expressed by mean and SD and were compared by the type of respondent by Student's $t$-test. The two-tailed significance level for all statistical tests was $P<0.05$. All analyses were carried out on SPSS software version 23.0 (SPSS, Inc., Chicago, IL).

Ethical aspects. All participants provided informed consent. Those participants who attended the seminars/courses gave their oral consent before the lectures. The study was approved by the Miguel Hernández University's Project Evaluation Committee (Ref: DMC.JRR.01.16).

\section{RESULTS}

Scale validation. Description of the study sample. Table 1 presents the characteristics of the study sample, which was made up of 283 students and residents in medicine and pharmacy, plus 66 potential aid workers. Once the elements with saturation in more than one factor or with factorial saturation of less than 0.50 were eliminated, the EFA converged into one dimension with 10 items (Table 2). The version of ChaLKS that emerged included the items that the experts had considered essential (Supplemental Table 4, 5a and b). The difficulty indexes ranged from 0.23 to 0.91 (Table 2). Seven of the 10 items showed an average difficulty index, and 1/10 was deemed moderately difficult. The item-total correlations ranged between 0.30 and 0.53 , and Cronbach's alpha was 0.75 . Just more than half $(54.6 \%)$ of the respondents

TABLE 1

Characteristics of all participants and the two samples of students and professionals in whom the scale was validated

\begin{tabular}{|c|c|c|c|c|c|c|}
\hline \multirow[t]{2}{*}{ Characteristic } & \multicolumn{2}{|c|}{ All participants } & \multicolumn{2}{|c|}{$\begin{array}{l}\text { Medicine and pharmacy } \\
\text { students and residents }\end{array}$} & \multicolumn{2}{|c|}{$\begin{array}{l}\text { Social and health } \\
\text { science }^{\star} \text { students }\end{array}$} \\
\hline & \multicolumn{2}{|c|}{349} & \multicolumn{2}{|c|}{$283(81.1)$} & \multicolumn{2}{|c|}{66 (18.9) } \\
\hline Age (years), mean (SD) & \multicolumn{2}{|c|}{$27(7.0)$} & \multicolumn{2}{|c|}{$28.9(7.0)$} & \multicolumn{2}{|c|}{$21(6.0)$} \\
\hline Studies & $N$ & $\%$ & $N$ & $\%$ & $N$ & $\%$ \\
\hline Medical students & 69 & 19.8 & 69 & 24.4 & - & - \\
\hline Pharmacy students & 14 & 4.0 & 14 & 4.9 & - & - \\
\hline Pharmacy residents & 18 & 5.2 & 18 & 6,4 & - & - \\
\hline Medical residents & 182 & 52.1 & 182 & 64.3 & - & - \\
\hline Specialist in family and community medicine & 82 & $23.5 \dagger$ & 82 & $45.1 \dagger$ & - & - \\
\hline Specialist in internal medicine and other specialties & 100 & $28.6 \dagger$ & 100 & $54.9 \dagger$ & - & - \\
\hline Other students & - & - & - & - & 66 & 18.9 \\
\hline Physiotherapy & 24 & 6.9 & - & - & 24 & $36.4 \dagger$ \\
\hline Occupational therapy & 15 & 4.3 & - & - & 15 & $22.7 \dagger$ \\
\hline Podiatry & 9 & 2.6 & - & - & 9 & 13.6† \\
\hline Psychology & 8 & 2.3 & - & - & 8 & $12.1 \dagger$ \\
\hline Nursing & 6 & 1.7 & - & - & 6 & $9.1 \dagger$ \\
\hline International relations & 2 & 0.6 & - & - & 2 & $3.0 \dagger$ \\
\hline Health technology & 2 & 0.6 & - & - & 2 & $3.0 \dagger$ \\
\hline Previous information on Chagas disease & 268 & 76.8 & 255 & 90.1 & 13 & 19.7 \\
\hline \multicolumn{7}{|l|}{ Recruitment events } \\
\hline Conferences on cooperation & 132 & 37.8 & 66 & 23.3 & 66 & 100 \\
\hline Master in infectious diseases & 47 & 13.5 & 47 & 16.6 & - & - \\
\hline Seminar on Chagas disease & 21 & 6.0 & 21 & 7.4 & - & - \\
\hline Course on care for immigrant patients & 82 & 23.5 & 82 & 29.0 & - & - \\
\hline Pre-congress workshop & 36 & 10.3 & 36 & 12.7 & - & - \\
\hline Online & 31 & 8.9 & 31 & 11.0 & - & - \\
\hline \multicolumn{7}{|l|}{ Place of birth } \\
\hline Spain & 313 & 89.7 & 251 & 88.7 & 62 & 93.9 \\
\hline Latin America & 25 & 7.2 & 25 & 8.8 & - & - \\
\hline Other European country & 9 & 2.6 & 6 & 2.1 & 3 & 4.5 \\
\hline Africa & 2 & 0.6 & 1 & 0.4 & 1 & 1.5 \\
\hline
\end{tabular}

†Percentage of the total medical residents/other students, respectively. 
TABLE 2

Results of the exploratory factor analysis and difficulty index for the ChaLKS items (10 items for ChaLKS-Medical and 8 for ChaLKS-Social\&Health)

\begin{tabular}{|c|c|c|c|c|}
\hline \multirow[b]{2}{*}{ Questionnaire items } & \multicolumn{2}{|c|}{ ChaLKS-Medical } & \multicolumn{2}{|c|}{ ChaLKS-Social\&Health } \\
\hline & Factorial saturation & Difficulty index & Factorial saturation & Difficulty index \\
\hline Is CD endemic to the Americas? & 0.81 & 0.91 & 0.79 & 0.20 \\
\hline Is CD endemic to Africa? & 0.80 & 0.84 & 0.71 & 0.20 \\
\hline Is CD endemic to Europe? & 0.74 & 0.91 & 0.87 & 0.32 \\
\hline Can CD be transmitted through blood transfusions? & 0.71 & 0.75 & - & - \\
\hline Can CD be transmitted from mother to child? & 0.70 & 0.76 & - & - \\
\hline Can CD be transmitted by kissing bugs? & 0.68 & 0.59 & 0.66 & 0.15 \\
\hline Can CD affect the heart? & 0.62 & 0.68 & 0.82 & 0.41 \\
\hline Can CD affect the stomach and bowels? & 0.62 & 0.65 & - & - \\
\hline Is CD diagnosed via serology? & 0.58 & 0.67 & - & - \\
\hline What drug is used to treat CD? & 0.53 & 0.23 & - & - \\
\hline Is there treatment for CD? & - & - & 0.80 & 0.33 \\
\hline $\begin{array}{l}\text { Can CD be transmitted by living with a person who } \\
\text { has the disease? }\end{array}$ & - & - & 0.79 & 0.23 \\
\hline \multirow[t]{3}{*}{ Can CD be transmitted through sexual contact? } & - & - & \multirow{2}{*}{\multicolumn{2}{|c|}{ Explained variance $59.86 \%$}} \\
\hline & \multicolumn{2}{|c|}{ Explained variance $46.66 \%$} & & \\
\hline & \multicolumn{2}{|c|}{ Cronbach's alpha 0.75} & \multicolumn{2}{|c|}{ Cronbach's alpha 0.89} \\
\hline
\end{tabular}

$\mathrm{CD}=$ Chagas disease ChaLKS $=$ Chagas Level of Knowledge Scale

answered seven or fewer items correctly; $16 \%$ obtained a perfect score.

Once the elements with saturation in more than one factor or with factorial saturation of less than 0.50 were eliminated, the EFA converged into one dimension with eight items. The difficulty indexes ranged from 0.15 to 0.41 (Table 2). Three of the eight items (37.5\%) showed an average difficulty index and $5 / 8(62.5 \%)$ a moderate one. The item-total correlations ranged between 0.47 and 0.79 . Cronbach's alpha was 0.89 . The vast majority $(87.1 \%)$ of the sample answered five or fewer questions correctly; $3.2 \%$ obtained a perfect score (Table 3).

Discriminant validity. The ChaLKS-Medical scores enabled good discrimination between knowledge among healthcare professionals and potential aid workers. The mean number of correct answers among nonmedical respondents was 1.8 (SD 2.4), compared with 7.0 (SD 2.3) among health professionals ( $t$-test 16.03, $P<0.001)$. The scores for the ChaLKSSocial\&Health also discriminated between the two main groups of respondents (nonmedical: 1.8 [SD 2.5] versus 6.9 [SD 1.6], $t$-test 20.2, $P<0.001$ ).

Knowledge of Chagas disease. Table 4 shows the itemized results for the ChaLKS-Medical and ChaLKS-Social\&Health questionnaire. The mean number of correct answers on the ChaLKS-Medical among healthcare students and residents was 7.0. Two difficult questions for respondents were as follows: "Can CD be transmitted through blood transfusions?" (67.1\% correct answers) and "Can CD be transmitted from mother to child?" (59.0\% correct answers). The most frequent mistake among health professionals and medical and pharmacy students related to the name of the drug used to treat $C D$ (23\% correct answers).

On the ChaLKS-Social\&Health scale, potential aid workers responded correctly to an average of 1.8 questions. The question garnering the most mistakes was "Can CD be transmitted from mother to child?" (15.2\% correct answers). Respondents also had little knowledge on the endemicity of the disease in the Americas or sexual transmission (19.7\% correct answers).

Table 5 presents the number of ChaLKS-Medical items yielding correct answers among healthcare professionals; those who had received information about CD in the past showed a better average performance (7.2 versus 4.3 correct answers; $P<0.001)$. This difference was apparent in nine of the 10 items analyzed (the exception was for the question "Can CD be transmitted from mother to child?"). The same

TABLE 3

Correct answers on the ChaLKS questionnaire: absolute, relative, and cumulative frequency

\begin{tabular}{|c|c|c|c|c|c|c|}
\hline \multirow[b]{2}{*}{ Correct answer } & \multicolumn{3}{|c|}{ ChaLKS-Medical } & \multicolumn{3}{|c|}{ ChaLKS-Social\&Health } \\
\hline & $N$ & Relative frequency & Cumulative frequency & $N$ & Relative frequency & Cumulative frequency \\
\hline 0 & 3 & 1.1 & 1.1 & 35 & 56.5 & 56.5 \\
\hline 1 & 7 & 2.5 & 3.5 & 5 & 8.1 & 64.5 \\
\hline 2 & 2 & 0.7 & 4.3 & 1 & 1.6 & 66.1 \\
\hline 3 & 13 & 4.6 & 8.9 & 5 & 8.1 & 74.2 \\
\hline 4 & 13 & 4.6 & 13.5 & 8 & 12.9 & 87.1 \\
\hline 5 & 26 & 9.2 & 22.7 & 0 & 0 & 87.1 \\
\hline 6 & 40 & 14.2 & 36.9 & 3 & 4.8 & 91.9 \\
\hline 7 & 50 & 17.7 & 54.6 & 3 & 4.8 & 96.8 \\
\hline 8 & 45 & 16 & 70.6 & 2 & 3.2 & 100 \\
\hline 9 & 38 & 13.5 & 84 & - & - & - \\
\hline 10 & 45 & 16 & 100 & - & - & - \\
\hline
\end{tabular}

ChaLKS = Chagas Level of Knowledge Scale; ChaLKS-Social\&Health = ChaLKS for potential aid workers in social and health fields. 
TABLE 4

Description and results of the questionnaire among the respondents to the ChaLKS-Medical $(n=283)$ and ChaLKS-Social\&Health $(n=66)$

\begin{tabular}{lcc}
\hline \multicolumn{1}{c}{ Item } & ChaLKS-Medical $(n=283)$ & ChaLKS-Social\&Health $(n=66)$ \\
\hline Correct answers, mean (SD) & $7.0(2.3)$ & $1.8(2.4)$ \\
Is CD endemic to the Americas? $n(\%)$ & $257(90.8)$ & $13(19.7)$ \\
Is CD endemic to Africa? $n(\%)$ & $184(65)$ & - \\
Is CD endemic to Europe? $n$ (\%) & $257(90.8)$ & $21(31.8)$ \\
Can CD be transmitted through blood transfusions? $n(\%)$ & $190(67.1)$ & - \\
Can CD be transmitted from mother to child? $n(\%)$ & $167(59.0)$ & $10(15.2)$ \\
Can CD be transmitted through sexual contact? $n(\%)$ & - & $13(19.7)$ \\
Can CD be transmitted by living with a person & & $15(22.7)$ \\
$\quad$ who has the disease? $n(\%)$ & - & $27(40.9)$ \\
Can CD be transmitted by an insect bite? $n(\%)$ & $239(84.8)$ & - \\
Can CD be transmitted by kissing bugs? $n(\%)$ & $213(75.3)$ & $13(19.7)$ \\
Can CD affect the heart? $n(\%)$ & $214(75.6)$ & - \\
Can CD affect the stomach and bowels? $n(\%)$ & - & - \\
Is CD diagnosed via serology? $n(\%)$ & $66(23.3)$ & $22(33.3)$ \\
Is there treatment for CD? $n(\%)$ & & - \\
What drug is used to treat CD? $n(\%)$ & & \\
\hline CD $=$ Chagas disease; ChaLKS $=$ Chagas Level of Knowledge Scale. &
\end{tabular}

occurred in the ChaLKS-Social\&Health questionnaire: those who had received information about CD in the past obtained better scores on the 8-item questionnaire (5.1 versus 1.1 correct answers; $P<0.001)$. This difference held for all items of the questionnaire.

We also compared responses among the 200 participating medical professionals and pharmacists (ChaLKS-Medical) according to whether they had been born and educated (until their bachelor's degree) in Latin America versus Europe (including Spain). The number of correct answers was similar in both groups; the only item showing differences was related to the endemicity of $C D$ in Africa, with Latin American professionals performing worse on this item than Europeans (39.2\% versus $76.3 \%$ correct answers; $P<0.001$; Supplemental Table 7).

\section{DISCUSSION}

The statistics applied to the validation analysis of these instruments confirm that both scales have a satisfactory factorial function, internal consistency, adequate difficulty indexes, and capacity to discriminate between different levels of knowledge of CD. Both instruments are appropriate for supporting aid actions in Chagas-endemic countries or in non-endemic countries providing health and social services to Latin American immigrants.

To our concern, and after reviewing the literature, there is only one mention of a validated survey about CD: a 22-item questionnaire, evaluated and validated through an initial pilot test involving 20 affected individuals and 20 health professionals. ${ }^{8}$ As advantages, we would like to highlight that our tool has been validated through a wider cohort of professionals, and also through students (from both social and healthcare fields), so its range of action is wider. In addition, ChaLKS-Medical and ChaLKS-Social\&Health have less items (10 and 8 items, respectively) so their use could be more operational.

In general, the level of knowledge shown in response to the questionnaires is adequate but could be improved. Our results were consistent with similar studies performed among physicians and medical students in non-endemic countries. $9,12,13,19$ Nevertheless, we drew conclusions based on results in convenience samples from selected populations with a special interest in global health, so our results are not generalizable. ${ }^{11}$ Moreover, we speculate that general awareness among physicians of other European countries (where CD is not as prevalent as in Spain) may be even lower. ${ }^{7}$

Some key aspects were well known among medical students and healthcare professionals, such as the endemicity of CD in Latin America. Nevertheless, some believed CD was also endemic to Africa, probably because they confused the CD parasite with the phylogenetically related parasite (also belonging to the genera Trypanosoma) responsible for African trypanosomiasis. Another epidemiologically crucial aspect was unknown among many healthcare professionals (up to 40\%): the mother-to-child transmission route. This is the most important transmission route in non-endemic countries where blood and organ donors are screened. Our results suggest that medical schools and postgraduate medical training should emphasize this aspect to a greater extent, especially in countries with Latin American populations.

Another aspect which respondents struggled with was the name of the drugs indicated for treating CD; less than $30 \%$ of the healthcare professionals and less than $9 \%$ of medical and pharmaceutical students knew the names. Nevertheless, we consider it much more important for professionals to know that a treatment exists than to be able to name the drug as such. In our previous pilot study conducted among medical students, $87 \%$ knew there was a specific treatment for CD, and around $75 \%$ were aware that most of the carriers of T. cruzi are asymptomatic. ${ }^{10}$ This issue is crucial to screen and encourage the population at risk to perform the diagnostic test. If health professionals are aware of the endemicity of the infection, its transmission routes (especially those at play in the country where the professional is based), its asymptomatic nature, which diagnostic test to order if CD is suspected, and the fact that treatment exists, we consider that the health professional is equipped with the basic knowledge to suspect, prevent, and treat CD.

The reactive item, "Can someone with CD feel okay (asymptomatic)?," which we consider relevant to discriminate the level of knowledge of CD, yielded a very low difficulty index (a high number of correct answers) and a low factorial saturation (0.29) in the analysis of construct validity. In light of these statistics, we chose not to retain the question in the ChaLKS. 


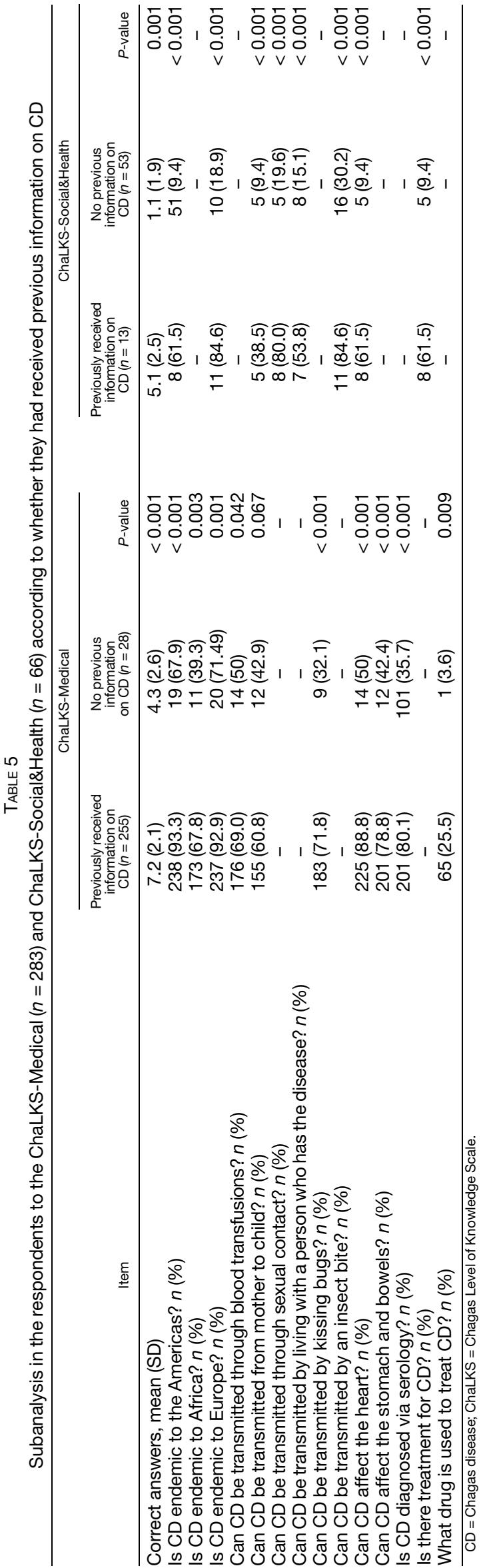

As pointed out in other articles, it is very important to measure the level of knowledge of NTDs in undergraduate medical students to evaluate the need to intensify education in these concepts, providing future medical doctors with optimal tools to cope with NTDs. ${ }^{20}$ We did not observe differences in the level of knowledge among resident physicians trained in Europe versus Latin America. In our opinion, this may reflect an acceptable training in imported diseases in Europe, equaling European medical students' CD knowledge tothe ones performing their bachelor's degree in endemic areas.

This study has some limitations. One is the low number of students who filled in the ChaLKS-Social\&Health. With regard to the items included on the scale, it would have been advisable to add a question on $C D$ transmission during organ transplantation to the initial version of the questionnaire to assess how it performed during the validation. Other knowledge items to consider assessing on future questionnaires would be the risk of stroke associated with CD (as some studies show the lack of knowledge on this aspect among health professionals $)^{21}$ and the existence of screening programs and activities to control the transmission of $T$. cruzi (regarding pregnant women in endemic areas, blood donations, and organ transplants) in the countries of the surveyed health professionals. ${ }^{3}$ This latter aspect would be advisable to include on both versions, given the importance of doctors having up-to-date information on these programs and of other health and social service providers knowing where to refer people who are at risk of carrying $T$. cruzi. Last, we controlled the index of difficulty of the questions, opting in most cases for questions with intermediate value indices. However, for some people, it may turn out that some questions are easier to answer than others and we cannot rule out the leading question effect in some cases.

The ChaLKS-Medical includes 10 items with good metric properties: 3 on epidemiology, 3 on transmission, 2 on clinical characteristics, 1 on diagnosis, and 1 on treatment. The question "Is CD endemic to the Americas?" shows high factorial saturation and a low difficulty index, so it could have been excluded from the questionnaire. However, the consulted experts considered this question too relevant to leave out, and it was thus maintained in the final version.

In the validation of the ChaLKS-Medical, pharmaceutical students and professionals were included because professionals from this sector may have a role in developing diagnostic methods and pharmacological treatments, as well as participating in multidisciplinary hospital teams that treat patients with $\mathrm{CD}^{22}$ In fact, pharmaceutical students have a yearly subject about parasitic diseases, including CD, within their bachelor's degree.

Chagas Level of Knowledge Scale-Social\&Health is an easier scale than ChaLKS-Medical, as its target audience has a different profile. This is why we excluded eight questions related to diagnosis and treatment, so ChaLKS-Social\&Health could focus on CD epidemiology and transmission. Regarding clinical manifestations of $C D$, we consider that, for volunteers and those studying health fields other than medicine and pharmacy, knowing about the cardiac implications of the disease is more important than understanding the involvement of the intestines. Cardiac involvement is the main cause of morbidity and mortality among patients infected with $T$. cruzi. In fact, $C D$ is the leading cause of cardiomyopathy and death due to cardiovascular disease in young people in Latin America. ${ }^{6}$ 
Our results show that potential aid workers who have previously received information on $C D$ have a higher level of knowledge of the disease, highlighting the desirability of providing enhanced training to this young collective. Approximately 500 aid programs in Latin America are launched from Spain every year. ${ }^{17}$ We believe that reinforcing knowledge about CD would be especially useful in the framework of these programs, as it would be beneficial for social and health aid workers aimed at Latin Americans-whether at home or abroad - to have knowledge of CD, regardless of whether the aid projects are directly related to this disease. Not taking this step would be a waste of a good opportunity: CD underdiagnosis remains very high ${ }^{5}$ and knowledge of CD in at-risk populations is low ${ }^{23}$; many people are affected by more than one NTD, ${ }^{24}$ and integrated programs seem to be effective toward reducing the burden of NTDs in low- and lowermiddle-income countries. ${ }^{25,26}$

Chagas Level of Knowledge Scale-Medical also showed a higher rate of correct answers among those who had received previous information on $\mathrm{CD}$, prompting a reflection on the need to continue improving training on CD in healthcare students and professionals, both in endemic and non-endemic countries. ${ }^{10,11,27}$ Awareness and a precise knowledge of CD in medical students, resident physicians, and healthcare professionals in general may facilitate an early diagnosis and correct clinical management in patients with $T$. cruzi infection and greater adherence to T. cruzi screening programs, ${ }^{28}$ also improving the overall healthcare for this population and the control of the disease. ${ }^{29}$ Future studies should assess the impact of these data on CD underdiagnosis, evaluating the need for educating students and healthcare professionals from non-endemic countries about this and other emerging diseases. ${ }^{19}$

$A$ validated questionnaire on knowledge about CD can be useful:

1. to use as a teaching tool before and after specific training sessions on CD for doctors, medical students, other health and social professionals, and potential aid workers;

2. to evaluate the level of knowledge among our health professionals and students in areas not endemic to CD, as a step to inform the design of training courses on NTDs and global health; and

3. to assess knowledge of CD in aid workers who wish to carry out their work in countries with vector-borne transmission of $C D$ or with Latin American migrants.

It would be desirable that similar initiatives are replicated in other countries and in relation to other NTDs to improve the training in global health among health professionals and aid workers.

Received September 11, 2019. Accepted for publication March 23, 2020.

Published online April 27, 2020.

Note: Supplemental information, tables, and figures appear at www.ajtmh.org.

Acknowledgments: We acknowledge Meggan Harris for proofreading the manuscript, Tania González Zivkovic for the forward-backward translation, and the consulted experts of Chagas disease for their valuable comments.

Authors' addresses: José M. Ramos-Rincón, Department of Clinical Medicine, Universidad Miguel Hernández de Elche, Alicante, Spain and Department of Internal Medicine, Hospital General Universitario de Alicante, Instituto de Investigación Sanitaria y Biomédica de Alicante (ISABIAL-Fundación FISABIO), Alicante, Spain, E-mail: jose.ramosr@goumh.umh.es. José J. Mira-Solves, Department of Health Psychology, Universidad Miguel Hernández de Elche, Campus de Elche, Alicante, Spain, and Alicante-Sant Joan Health District, Alicante, Spain, E-mail: juanjose.mira@umh.es. Violeta RamosSesma, Department of Internal Medicine, Hospital Universitario de Torrevieja, Alicante, Spain, E-mail: vramossesma@gmail.com. Diego Torrús-Tendero, Department of Internal Medicine, Hospital General Universitario de Alicante, Instituto de Investigación Sanitaria y Biomédica de Alicante (ISABIAL-Fundación FISABIO), Alicante, Spain, and Parasitology Area, Universidad Miguel Hernández de Elche, Alicante, Spain, E-mail: torrus_die@gva.es. Jara Llenas-García, Department of Internal Medicine, Hospital Vega Baja, Fundación para el Fomento de la Investigación Sanitaria y Biomédica de la Comunitat Valenciana (FISABIO), Orihuela, Spain, E-mail: jarallenas@gmail.com. Miriam Navarro, Department of Public Health, Science History and Gynecology, Universidad Miguel Hernández de Elche, Alicante, Spain, E-mail: miriamtropical@gmail.com.

\section{REFERENCES}

1. Khamisy-Farah R, Adawi $M$, Jeries-Ghantous $H$, Bornstein J, Farah R, Bragazzi NL, Odeh M, 2019. Knowledge of human papillomavirus (HPV), attitudes and practices towards anti-HPV vaccination among Israeli pediatricians, gynecologists, and internal medicine doctors: development and validation of an ad hoc questionnaire. Vaccines (Basel) 7: E157.

2. Diniz KKS, Pagano AS, Fernandes APPC, Reis IA, Pinheiro Júnior LG, Torres HC, 2019. Development and validation of an instrument to assess Brazilian healthcare professional providers' knowledge on sickle cell disease. Hematol Transfus Cell Ther 41: 145-152.

3. Requena-Méndez A, Albajar-Viñas $P$, Angheben $A$, Chiodini $P$, Gascón J, Muñoz J; Chagas Disease COHEMI Working Group, 2014. Health policies to control chagas disease transmission in European countries. PLoS Negl Trop Dis 8: e3245.

4. Ramos JM et al., 2012. Multicenter epidemiological and clinical study on imported chagas diseases in Alicante, Spain. Pathog Glob Health 106: 340-345.

5. Basile L et al., 2011. Chagas disease in European countries: the challenge of a surveillance system. Euro Surveill 16: 19968.

6. Pérez-Molina JA, Molina I, 2018. Chagas disease. Lancet 391: 82-94.

7. Navarro $M$ et al., 2017. Cross-sectional, descriptive study of Chagas disease among citizens of Bolivian origin living in Munich, Germany. BMJ Open 7: e013960.

8. Claveria Guiu I, Caro Mendivelso J, Ouaarab Essadek H, González Mestre MA, Albajar-Viñas P, Gómez I Prat J, 2018. The catalonian expert patient programme for Chagas disease: an approach to comprehensive care involving affected individuals. J Immigr Minor Heal 19: 80-90.

9. Muñoz-Vilches MJ, Salas-Coronas J, Gutiérrez-Izquierdo MI, Metz D, Salvador-Sánchez J, Giménez-Sánchez F, 2013. Health professionals' knowledge on Chagas disease in the province of Almeria, Spain. Rev Esp Salud Publica 87: 267-275.

10. Ramos JM, Martorell C, López-Amorós Al, Navarro M, 2017. ¿Cuánto saben los estudiantes del Grado en Medicina sobre la enfermedad de Chagas en España? Gac Sanit 31: 171-172.

11. Roger D, González-Escalada ANM, 2017. Evaluación del conocimiento sobre Enfermedades Tropicales Desatendidas en estudiantes de sexto curso del grado en Medicina de la Comunidad Autónoma de Madrid. Bilbao, Spain, 23-25 October 2017. Bilbao, Spain: X National Congress of the Spanish Society of Tropical Medicine and International Health.

12. Verani JR, Montgomery SP, Schulkin J, Anderson B, Jones JL, 2010. Survey of obstetrician-gynecologists in the United States about Chagas disease. Am J Trop Med Hyg 83: 891-895.

13. Amstutz-Szalay S, 2017. Physician knowledge of Chagas disease in hispanic immigrants living in appalachian Ohio. $J$ Racial Ethn Heal Disparities 4: 523-528.

14. Nunes MCP et al.; American Heart Association Rheumatic Fever, Endocarditis and Kawasaki Disease Committee of the Council on Cardiovascular Disease in the Young; Council on 
Cardiovascular and Stroke Nursing; Stroke Council, 2018, Chagas cardiomyopathy: an update of current clinical knowledge and management: a scientific statement from the American heart association. Circulation 138: e169-e209.

15. Iglesias-Rus L, Romay-Barja M, Boquete $T$, Benito A, BlascoHernández T, 2019. The role of the first level of health care in the approach to Chagas disease in anon-endemic country. PLoS Negl Trop Dis 13: e0007937.

16. Pinazo M-J et al., 2017, A strategy for scaling up access to comprehensive care in adults with Chagas disease in endemic countries: the Bolivian Chagas Platform. PLoS Negl Trop Dis 11: e0005770.

17. Portal Web AECID América Latina y Caribe [Internet]. [cited 19 Jun 2019]. Available at: http://www.aecid.es/ES/dónde-cooperamos/ alc. Accessed March 11, 2019.

18. Pérez de Ayala Balzola A, Pérez-Molina JA, Navarro Beltrá M, López-Vélez R, 2009. Enfermedad de Chagas en personas procedentes de Latinoamérica residentes en España. Ministerio de Sanidad y Política Social. Available at: https:// www.mscbs.gob.es/profesionales/saludPublica/prevPromocion/ promocion/migracion/docs/enfermedadChagas.pdf. Accessed December 4, 2019.

19. Stimpert KK, Montgomery SP, 2010. Physician awareness of Chagas disease, USA. Emerg Infect Dis 16: 871-872.

20. Errea RA et al., 2015. Medical student knowledge of neglected tropical diseases in Peru: a cross-sectional study. PLoS Negl Trop Dis 9: e0004197.

21. Carod-Artal FJ, Ribeiro Lda S, Vargas AP, 2007. Awareness of stroke risk in chagasic stroke patients. J Neuro/ Sci 263: 35-39.
22. Romo ML, 2014. Need for pharmacist awareness of Chagas disease. Am J Health Syst Pharm 71: 1069-1070.

23. Navarro M, Perez-Ayala A, Guionnet A, Perez-Molina JA, Navaza B, Estevez L, Flores-Chavez M, Lopez-Velez R, 2011. Targeted screening and health education for Chagas disease tailored to at-risk migrants in Spain, 2007 to 2010. Euro Surveill 16: 19973.

24. Hotez PJ, Bottazzi ME, Franco-Paredes C, Ault SK, Periago MR, 2008. The neglected tropical diseases of Latin America and the Caribbean: a review of disease burden and distribution and a roadmap for control and elimination. PLoS Negl Trop Dis 2: e300.

25. Stolk WA, Kulik MC, le Rutte EA, Jacobson J, Richardus JH, de Vlas SJ, Houweling TA, 2016. Between-country inequalities in the neglected tropical disease burden in 1990 and 2010, with projections for 2020. PLoS Negl Trop Dis 10: e0004560.

26. Barogui YT et al., 2018. Integrated approach in the control and management of skin neglected tropical diseases in Lalo, Benin. PLoS Negl Trop Dis 12: e0006584.

27. Lugo-Caballero Cl, Dzul-Rosado K, Dzul-Tut I, Balam-May Á, Zavala-Castro J, 2017. Knowledge of vector-borne diseases (dengue, rickettsiosis and Chagas disease) in physicians. Gac Med Mex 153: 321-328.

28. Ramos JM, Milla A, Rodríguez JC, López-Chejade P, Flóres M, Rodríguez JM, Gutierrez F, 2012. Chagas disease in Latin American pregnant immigrants: experience in a non-endemic country. Arch Gynecol Obstet 285: 919-923.

29. Berger BA, Bartlett AH, Jiménez-Hernández R, Trinidad Vázquez E, Galindo-Sevilla N, 2018. Physician knowledge, attitudes, and practices related to Chagas disease in tabasco, Mexico. Am J Trop Med Hyg 98: 1743-1747. 